\title{
Role of Nucleated Red Blood Cells in Diagnosis of Neonatal Asphyxia in Neonate with Meconium Stained and Clear Amniotic Fluid Ali El Shabrawy Ali ${ }^{1}$, Naglaa Ali Ali Khalifa ${ }^{2}$, Ihab Abd El-Moniem El-Bana ${ }^{3}$, Fatma Medhat Abdel-salam Ali*1
}

Departments of ${ }^{1}$ Obstetrics and Gynecology, ${ }^{2}$ Clinical Pathology and

${ }^{3}$ Pediatric and Neonatology, Faculty of Medicine, Zagazig University, Egypt

*Corresponding Author: Fatma Medhat Abdel-salam Ali, Mobile: (+20)01061135467, Email: dr.euroka@ yahoo.com

\begin{abstract}
Background: Perinatal asphyxia has been defined as the lack of oxygen that occurs either before, during, or after birth. Many studies in recent past have suggested that an increased number of nucleated red blood cells (NRBC) in umbilical cord blood may be a useful marker to identify birth asphyxia.

Objective: To find out the relationship between meconium-stained amniotic fluid and NRBC in umbilical cord blood. Patients and Methods: This was a comparative cross-section study, which was carried out at Obstetrics and Gynecology Department, Zagazig University Hospitals on 54 cases divided into 2 groups: (Group 1): included 27 pregnant females with meconium-stained amniotic fluid who gave birth to neonates (male 15 and female12) showing criteria of perinatal asphyxia, (Group 2): included 27 pregnant females with clear amniotic fluid who gave birth to neonate's apparently healthy full-term (male17 and female10) as control group with no obstetrical problems.

Results: There was no statistically significant difference between case and control groups in basic characteristics or obstetric history. NRBC's/100 WBCs and lactate were highly significantly increased in group 1 . The mean value of lactate was increasing in relation to stage of hypoxic ischemic encephalopathy (HIE).

Conclusion: There was a strong correlation between markers of acute asphyxia (i.e., umbilical artery blood nucleated red blood cells and lactate,) and meconium-stained liquor cases. These markers were significantly elevated in newborns with meconium-stained amniotic fluid.
\end{abstract}

Key words: Amniotic fluid, Lactate, Neonatal Asphyxia, Nucleated Red Blood Cells, Stained.

\section{INTRODUCTION}

Perinatal asphyxia is a common and serious neonatal problem and contributes to both neonatal morbidity and mortality. According to the World Health Organization (WHO) in 2000, of the 130 million infants born globally each year, approximately 4 million babies die before they reach the age of 1 month. Furthermore, of 1.2 million neonatal deaths in India, 300,000-350,000 babies die due to perinatal asphyxia mostly within first 3 days of life ${ }^{(1)}$.Hypoxic ischemic encephalopathy (HIE) after prenatal asphyxia is an important cause of neonatal morbidity, neurological disability and mortality. The early prediction of hypoxic ischemic encephalopathy is particularly important because of the brief therapeutic window and possible side effects of neuroprotective intervention ${ }^{(2)}$.

Meconium-stained amniotic fluid is common during labor and affects $10-15 \%$ of neonates. Approximately $5 \%$ of these neonates develop meconium aspiration syndrome (MAS). Despite advances in neonatal resuscitation and respiratory care, MAS remains a serious cause of neonatal mortality and morbidity, especially in developing countries and lowresource settings. It is generally accepted that activation of colonic peristalsis triggered by fetal hypoxiaischemia leads to the passage of meconium and fetal gasping movements, resulting in meconium aspiration and respiratory distress ${ }^{(3)}$.
Nucleated red blood cells (NRBCs) are commonly found in the cord blood of the newborn infants. Various situations may contribute to elevated level of NRBC such as prematurity, maternal diabetes mellitus, fetal anemia, growth retardation and $\mathrm{Rh}$ sensitization ${ }^{(4)}$.

Nucleated red blood cells count per 100 white blood cells in umbilical venous blood of newborn has been reported as a simple, quick and cheap marker of prenatal asphyxia, based on the fact that hypoxic events induce fetal compensatory response in the form of exaggerated erythropoiesis and influx of immature red blood cells fetal circulation ${ }^{(5)}$.

Lactate is produced in the event of hypoxia and poor tissue perfusion. Any reduction of oxygen and substrate delivery to the fetus, aerobic metabolism through Krebs cycle cannot be sustained and tissue need anaerobic metabolism to meet energy requirement, this in turn leads to increase in the production and accumulation of blood lactate ${ }^{(\boldsymbol{(})}$.

Blood lactate concentration in critically ill and injured patients can be used to detect tissue hypoxia at an early stage which is simple, cheap and quick marker to predict and asses illness severity and outcome ${ }^{(7)}$.

The objective of this study was to find out the relationship between meconium stained amniotic fluid and NRBC in umbilical cord blood. 


\section{PATIENTS AND METHODS}

This was a comparative cross-section study, which was carried out at Obstetrics and Gynecology Department, Zagazig University Hospitals on 54 cases divided into 2 groups: Group (1): included 27 pregnant females with meconium-stained amniotic fluid who gave birth to neonates (male 15 and female 12) that showed criteria of perinatal asphyxia, and Group (2): included 27 pregnant females with clear amniotic fluid who gave birth to neonate's apparently healthy full-term (male 17 and female 10) as control group without obstetrical problems, during period from February 2020 till August 2021.

Inclusion criteria: Pregnant women who delivered singleton live-born infants of 37 to 42 completed weeks' gestation with turbid and clear amniotic fluid by sonar without any complications during pregnancy were included in the study, and all pregnant women that were diagnosed by amniotic fluid turbidity (meconium) during antenatal period by Doppler ultrasound or CTG (cardiotocography) and decisions took in those cases.

Exclusion criteria: Preterm $(<37$ weeks of gestational age), babies with congenital heart disease, babies with $\mathrm{Rh}$ isoimmunisation, babies with chromosomal anomalies if diagnosed early, multiple pregnancies, women with diabetes, hypertension, preeclampsia, and chorioamnionitis, smoking during pregnancy, previous low birth weight infants, or infants with cyanotic disease were excluded from the study.

Criteria of perinatal asphyxia: (1) Profound metabolic or mixed acidemia $\mathrm{pH}<7$ in an umbilical cord arterial blood sample. (2) Persistence of an Apgar score of 0 to 3 at 5 minutes. (3) Clinical neurologic squeal in the immediate neonatal period (e.g. seizures, hypotonia, coma or hypoxic ischemic encephalopathy). (4) Evidence of multiorgan system dysfunction in the immediate neonatal period.

All patients were subjected to the following:

A-Full history taking from parents including:

1. Chief Complaint: Brief statement of primary problem (including duration) that caused family to seek medical attention.

2. History of maternal present illness.

3. Pregnancy and Birth History.

4. Review of Neonatal Systems.

5. Family History.

B- Complete clinical examination:

C- Investigations:

Complete general examination.

Laboratory work: collection of blood sample:

- After obtaining $2 \mathrm{ml}$ of umbilical cord blood in a sterile syringe, during resuscitation, it was transferred within 30 minutes in an EDTA tube to the Clinical Pathology Department. The blood was fixed with ethanol and peripheral smear was obtained with Leishman stain on a glass slide. The number of nucleated RBCs for 100 WBCs were observed and noted by the clinical pathologist.

- Collected TLC (total leuckocyte count) $\equiv$ $\frac{\text { TLC }}{100+\text { normal count }} \times 100$.

- EX: TLC $=15000$, Normoblast $=50 / 100 \mathrm{WBC}_{\mathrm{S}}$

- Collected TLC $=\frac{15000}{100+50} \times 100=10000$

- Routine investigations: e.g.: Doppler ultrasound and CTG (cardiotocography).

Laboratory: Complete blood picture by automated cell counter (xn330), and serum lactate by automated autoanalyzer COBAS 6000c.

\section{Ethical consent:}

An informed consent from parents of the participants was taken and confidentiality of information was assured. An official written administrative permission letter was obtained from dean of Faculty of Medicine, Zagazig University, Head of Obstetrics and Gynecology Department and Head of Pediatric and Neonatology Department, in the same university. The title and objectives of the study were explained to them to ensure their cooperation. This work has been carried out in accordance with The Code of Ethics of the World Medical Association (Declaration of Helsinki) for studies involving humans.

\section{Statistical analysis}

The collected data were coded, processed and analyzed using the SPSS (Statistical Package for the Social Sciences) version 22 for Windows ${ }^{\circledR}$ (IBM SPSS Inc, Chicago, IL, USA). Data were tested for normal distribution using the Shapiro Wilk test. Qualitative data were represented as frequencies and relative percentages. Chi square test $\left(\chi^{2}\right)$ was used to calculate difference between two or more groups of qualitative variables. Quantitative data were expressed as mean \pm SD (Standard deviation). Independent samples t-test was used to compare between two independent groups of normally distributed variables (parametric data). Receiver operating characteristic curve (ROC) was used to permit the selection of threshold values and comparison of different test strategy allowing the calculation of sensitivity and specify positivity and negativity. $\mathrm{P}$ value $<0.05$ was considered significant.

\section{RESULTS}

This table shows no statistically significant difference between case and control groups in basic characteristic and obstetric history (Table 1). 
Table (1): Basic characteristics and obstetric history of the studied groups ( $n=54)$

\begin{tabular}{|c|c|c|c|}
\hline Variables & $\begin{array}{c}\text { Group1 } \\
(\mathbf{n}=27)\end{array}$ & $\begin{array}{c}\text { Group2 } \\
(n=27)\end{array}$ & $\mathbf{P}$ \\
\hline Age (years): Mean \pm SD & $27.4 \pm 6.6$ & $28.3 \pm 4.9$ & 0.4 \\
\hline Gestational age at delivery (weeks): Mean \pm SD & $38.8 \pm 1.2$ & $39.0 \pm 2.7$ & 0.07 \\
\hline $\begin{array}{ll}\text { Gravidity: } & \\
& \text { Primigravida } \\
& \text { Multigravida }\end{array}$ & $\begin{array}{c}3(11.5 \%) \\
24(88.5 \%)\end{array}$ & $\begin{array}{c}5(17.3 \%) \\
22(82.7 \%)\end{array}$ & 0.4 \\
\hline $\begin{array}{l}\text { Nullipara } \\
\text { Primipar } \\
\text { Multipara }\end{array}$ & $\begin{array}{c}6(21.2 \%) \\
4(17.3 \%) \\
17(61.5 \%)\end{array}$ & $\begin{array}{c}5(19.2 \%) \\
3(13.5 \%) \\
19(67.3 \%)\end{array}$ & 0.7 \\
\hline
\end{tabular}

This table shows that there was no significant difference as regard GA, birth weight, sex distribution and no relation with development of neonatal asphyxia (Table 2).

Table (2): Clinical characteristics of the neonates between studied groups

\begin{tabular}{|l|l|l|c|c|c|}
\hline \multicolumn{2}{|l|}{} & Group 1 (n = 27) & Group 2 (n = 27) & P \\
\hline \multicolumn{3}{|l|}{ Birth weight (g) } & $3605.25 \pm 310.5$ & $3790.0 \pm 278.90$ & 0.055 \\
\hline \multirow{3}{*}{ Sex } & Male & $\mathrm{N}$ & 15 & 17 & \\
\cline { 2 - 6 } & $\%$ & $60.0 \%$ & $65.0 \%$ & \\
\cline { 2 - 6 } & Female & $\mathrm{N}$ & 12 & 10 & 0.74 \\
\cline { 2 - 6 } & & $40.0 \%$ & $35.0 \%$ & \\
\hline
\end{tabular}

This table shows that NRBC's/100 WBCs were highly significantly increased in case group 1 (Table 3).

Table (3): NRBC's/100 WBCs distribution in studied groups

\begin{tabular}{|l|c|c|c|}
\hline \multicolumn{1}{|c|}{ NRBC's/100 WBCs } & Group 1 & Group 2 & P \\
\hline Mean & $\mathbf{1 8 . 6 3}$ & 5.87 & $<0.001$ \\
\hline SD & 4.63 & 1.06 & $<0.001$ \\
\hline
\end{tabular}

This table shows that lactate was highly significantly increased in case group 1 (Table 4).

Table (4): Lactate level distribution in studied groups

\begin{tabular}{|l|c|c|c|}
\hline \multicolumn{1}{|c|}{ Lactate (mmol/L) } & Group 1 & Group 2 & P \\
\hline Mean & $\mathbf{5 . 6 3}$ & 3.11 & $<0.001$ \\
\hline SD & 1.63 & 0.6 & $<0.001$ \\
\hline
\end{tabular}

Lactate was $100 \%$ specific and 88.9 sensitive in early detection of neonatal asphyxia in cases with meconium stained fluid (Table 5).

Table (5): Diagnostic criteria of lactate to predict hypoxic ischemic encephalopathy (HIE) cases

\begin{tabular}{|l|c|c|c|c|c|}
\hline & Cut off & Sensitivity & specificity & NPV & PPV \\
\hline Lactate (mmol/L) & $>5.1$ & 88.9 & 100 & 98.0 & 93.3 \\
\hline
\end{tabular}

NPV: Negative predictive value, PPV: Positive predictive value

NRBCs is $95 \%$ specific and 85 sensitive in early detection of neonatal asphyxia in cases with meconium stained fluid (Table 6).

Table (6): Diagnostic criteria of NRBCs to predict hypoxic ischemic encephalopathy (HIE) cases

\begin{tabular}{|c|c|c|c|c|c|}
\hline & Cut off & Sensitivity & specificity & NPV & PPV \\
\hline NRBCs & $(>6.5 \mathrm{NRBCs} / 100 \mathrm{WBC})$ & 85 & 95 & 96.0 & 92.3 \\
\hline
\end{tabular}

\section{DISCUSSION}

There was no statistically significant difference between case and control groups as regard age, gestational age at delivery, gravidity and parity. There was no significant difference regarding birth weight and sex distribution among the studied groups. In accordance with our results, the study of Elsokkary $\boldsymbol{e t}$

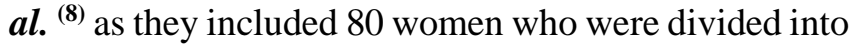
2 groups: Group I $(\mathrm{n}=40)$ : included women with meconium-stained amniotic fluid. Group II $(n=40)$ : included women with clear liquor. There were no statistically significant differences both groups concerning demographic data (maternal age, gestational, parity, and birth weight and sex distribution). Similarly, Badr et al. (9) showed the population characteristics of the two groups of babies: those born hypoxic ischemic encephalopathy and normal babies. They were comparable in terms of gestational age, birth weight and gender distribution. 
The current study showed that there was no significant difference as regard mode of delivery. However, in the study of Einikyte $\boldsymbol{e t}$ al. ${ }^{(6)}$, there was a significant difference in group 1 compared with group 2 and operative vaginal deliveries were performed as follows: $(n=10,25.6 \%$ versus $n=32,3.7 \%$; $p<0.001)$. Women in group 1 also underwent more caesarean sections $(\mathrm{n}=18,46.2 \%$ versus $\mathrm{n}=209,24.2 \% ; \mathrm{p}=$ 0.004). While, in the study of Shivaprakash and Nigam ${ }^{(10)}$, there is higher incidence of instrumental delivery in cases compared to controls but no significant difference in LSCS.

In the study in our hands, no significant differences between studied groups in WBC and $\mathrm{Hb}$. NRBC's/100 WBCs was highly significantly increased in group 1. Our results were in agreement with study of Elsokkary $\boldsymbol{e t}$ al. ${ }^{(\mathbf{8})}$ as they reported that there were no statistically significant differences concerning cord blood WBC count and hemoglobin level. However, there were statistically highly significant differences concerning NRBC per $\mathrm{mm}^{3}$ and NRBC per $100 \mathrm{WBCs}$ with higher values in the MSAF group. There was also a higher abnormal NRBC percentage $(\geq 10 \%)$ in the MSAF group. As well, Papa et al. ${ }^{(11)}$ conducted a prospective comparative study to the cord blood nucleated RBC count (NRBC) in asphyxiated and nonasphyxiated fetuses at birth to find out the correlation between NRBC count, fetal acidosis and the clinical markers of asphyxia. They studied 51 cases with nonasphyxiated fetuses and 52 asphyxiated fetuses. They found that the mean NRBC count in the study group was $25.65 \pm 10.14$ as compared to $12.33 \pm 5.51$ in the control group ( $\mathrm{p}=0.003)$. They concluded that NRBC count correlates well with fetal acidosis in asphyxiated neonates. Similar observation was made by Darkhaneh et al. ${ }^{(12)}$. Moreover, Kanodi et al. ${ }^{(13)}$ stated that out of the 82 neonates in case group, fiftynine (59) neonates were found to have NRBC level $\geq 10 / 100 \mathrm{WBC}$, out of which $58(70.7 \%)$ were cases and $1(1.2 \%)$ was a control. NRBCs count of $\geq 10 / 100 \mathrm{WBC}$ was seen more in the newborn who had low 5 min Apgar score and in the newborn with severe HIE, these association were statistically significant $(\mathrm{P}$ value; 0.001).

The present study showed that the mean value of lactate was increasing in relation to stage of HIE. Using ROC curve for lactate to predict HIE cases: the cutoff point was $>5.1$, the sensitivity was $88.9 \%$, the specificity was $100 \%$, NPV was $98 \%$ and PPV was $93.3 \%$. This result goes hand in hand with the results of the study of Haiju et al. ${ }^{(14)}$ who conduct a study to establish a simple and quick method that could be used to predict the occurrence of hypoxic ischemic encephalopathy (HIE) as early as possible by investigating the variations of nucleated red blood cells per 100 white blood cell (NRBC/100 WBC) counts and lactate levels in cord blood. They measured the percentage (NRBCs/100 WBC) and lactate in the umbilical blood in 46 cases of acute fetal distress
(AFD) and 54 cases of chronic fetal distress (CFD) neonates. They reported that both lactate levels and NRBC/100 WBC counts were higher in CFD and AFD groups than in controls (both $\mathrm{P}<0.01$ ).

In the study of Chiang et al. ${ }^{(15)}$, they showed that serum level of lactate $(\mathrm{mg} / \mathrm{dL})$ after 72 hours of $\mathrm{TH}$ (therapeutic hypothermia) was higher (35.6 vs. 13.8, $\mathrm{p}$ $=0.042$ ) in infants with PND (poor neurodevelopment) comparing with infants without PND at 24 months of age. Also, Van Anh et al. ${ }^{(16)}$ revealed that umbilical cord blood lactate level to diagnose HIE was $>8.12$ $\mathrm{mmol} / \mathrm{L}$, which yielded a sensitivity of $73.7 \%$, and specificity of $86.4 \%$. Overall accuracy was good, with area under ROC curve of 0.799 (95\% CI: 0.645-0.908), $\mathrm{p}=0.0001$. This is in agreement with some studies, which concluded that umbilical lactate can be used in a middle-low resource setting as a measurement of intrapartum hypoxia, with reasonable sensitivity and specificity. Elfarargy et al. ${ }^{(17)}$ reported that serum lactate level to diagnose $\mathrm{HIE}$ was $>3.6 \mathrm{mmol} / \mathrm{L}$, which yielded a sensitivity of $87 \%$, specificity $100 \%$, PPV $100 \%$ and NPV $88 \%$ with a diagnostic accuracy of $93 \%$.

Finally, our results showed that the mean value of NRBC's/100 WBC count was increasing in relation to stage of HIE. Using ROC curve for NRBCs to predict HIE: the cutoff point was (>6 .5 NRBCs/100 WBC), the sensitivity was $85 \%$, the specificity was 95\%, NPV was $96 \%$ and PPV was $92.3 \%$. Our results were supported by study of Elfarargy et al. ${ }^{(17)}$ as they reported that there was a highly significant difference between both groups in relation to nucleated red blood cells (higher in HIE than in control) (P value < 0.001). There was highly significant difference in outcome among different grades of hypoxia (P value $<0.001)$. It also showed significant difference between levels of nucleated red blood cells ( $\mathrm{P}$ value $<0.001$ ). The best cutoff of nucleated red blood cells to detect hypoxia was > 4.5/100 WBCs with a sensitivity of $77 \%$, specificity $83 \%$, PPV $82 \%$ and NPV $78 \%$ with a diagnostic accuracy of $80 \%$.

This is in concordance with some studies held by Li et al. ${ }^{(18)}$ who stated that NRBC counts can predict brain injury and neurological outcomes in asphyxiated neonates. Also, Kanodi et al. ${ }^{(13)}$ revealed that the cut off NRBC value of $\geq 10 / 100 \mathrm{WBC}$ also found to have a sensitivity of $70.30 \%$ with a specificity of $98.78 \%$. NRBC has a positive predictive value of $98.31 \%$ with a negative predictive value of $77.14 \%$. Significance and sensitive area for ROC curve was 0.875 . The ROC curve was calculated with cut off NRBC value of $\geq 10 / 100$ WBC. They concluded that NRBC counts can be very useful to differentiate HIE newborns from nonHIE newborns, which will help in appropriate management and better outcome of these newborns.

In the study of Boskabadi et al. ${ }^{\left({ }^{19)}\right.}$, they showed a statistically significant relationship between NRBC/100 WBC and absolute NRBC count levels and the outcome of asphyxiated neonates. NRBC count 
more than 11 per $100 \mathrm{WBC}$, had sensitivity of $85 \%$ and specificity of $90 \%$ in predicting complications of asphyxia. Also, absolute NRBC count more than 1554, had sensitivity of $85 \%$ and specificity of $87 \%$ in predicting complications of asphyxia. Furthermore, Goel et al. ${ }^{(20)}$ found that higher the HIE staging; higher was the mean NRBC/100 WBC. It was observed that in HIE stage 1 mean NRBC/100 WBC was $10.17 \pm 2.64$. It was $19.04 \pm 6.86$ and $45.14 \pm 32.41$ for stages II and III respectively. Increase in NRBC count as marker for fetal asphyxia in their study had a sensitivity of $94 \%$, specificity of $98 \%$, positive predictive value of $98 \%$, negative predictive value of $94 \%$ with an overall accuracy of $96 \%$.

\section{CONCLUSION}

There was a strong correlation between markers of acute asphyxia (i.e., umbilical artery blood nucleated red blood cells, lactate) and meconium stained liquor cases. These markers were significantly elevated in newborns with meconium-stained amniotic fluid. The cut off value of lactate was $>5.1$ in case group with meconium stained amniotic fluid while NRBC/100WBC cut off value was $>6.5$. The nucleated red blood cell count and lactate level at birth not only reflects a response of the infant to perinatal hypoxia but is also a reliable index of early perinatal brain damage.

\section{Financial support and sponsorship: Nil.}

Conflict of interest: Nil.

\section{REFERENCES}

1. Kawakami M, Sanudo A, Teixeira M et al. (2021): Neonatal mortality associated with perinatal asphyxia: a population-based study in a middle-income country. BMC Pregnancy Childbirth, 21: 169-174.

2. Bano S, Chaudhary V, Garga U (2017): Neonatal hypoxic-ischemic encephalopathy: A radiological review. J Pediatr Neurosci., 12(1):1-6.

3. Yokoi K, Iwata O, Kobayashi $\mathrm{S}$ et al. (2021): Evidence of both fetal inflammation and hypoxiaischemia is associated with meconium aspiration syndrome. Sci Rep., 11: 1-7.

4. Ahearne C, Boylan G, Murray D (2016): Short- and long-term prognosis in perinatal asphyxia: An update. World Journal of Clinical Pediatrics, 5(1): 67-73.

5. Poryo M, Wissing A, Aygün A et al. (2017): Reference values for nucleated red blood cells and serum lactate in very and extremely low birth weight infants in the first week of life. Early Human Development, 105: 49-55.

6. Einikyte R, Snieckuviene V, Ramasauskaite $D$ et al. (2017): The comparison of umbilical cord arterial blood lactate and $\mathrm{pH}$ values for predicting short-term neonatal outcomes. Taiwanese Journal of Obstetrics and Gynecology, 56(6): 745-749.

7. Akerman $F$, Mokarami $P$, Källén et al. (2018): The small-for-gestational-age fetus has an intact ability to develop lacticemia when exposed to hypoxia: a retrospective comparative register study. Journal of
Maternal-Fetal and Neonatal Medicine, 31 (10): 12901297.

8. Elsokkary M, Mamdouh A, Nossair W et al. (2019): Significance of assay of nucleated RBCs in umbilical cord blood in neonates with meconium-stained amniotic fluid. The Journal of Maternal-Fetal \& Neonatal Medicine, 32(3): 483-487.

9. Badr M, Ali Y, Abdelhady M et al. (2011): Urinary lactate/creatinine ratio as early predictor of hypoxic ischemic encephalopathy in term neonates admitted to NICU, Zagazig University Hospitals. Research Journal of Medicine and Medical Sciences, 6(2): 54-61.

10. Shivaprakash N, Nigam G (2013): Prediction of HIE by nucleated RBC's in cord blood, serum creatine kinase and assessment of outcome by follow up upto 6 months. Journal of Evolution of Medical and Dental Sciences, 2(19): 3340-3349.

11. Papa D, Jyotsna G, Ashok B (2008): Cord blood nucleated red blood cell count a marker of fetal asphyxia. J Obstet Gynecol India, 58: 45-48.

12. Darkhaneh R, Ghanbari A, Asgharnia $M$ et al. (2013): Comparison of nucleated red blood cells in the umbilical cord of term neonates in healthy women and women with preeclampsia. Iran J Reprod Med., 11(1): 25-30.

13. Kanodi B, Bhatta N, Singh R et al. (2015): Nucleated red blood cell in cord blood as a marker of perinatal asphyxia. Journal of Nepal Paediatric Society, 35(3): 14.

14. Haiju Z, Suyuan H, Xiufang F et al. (2008): The combined detection of umbilical cord nucleated red blood cells and lactate: early prediction of neonatal hypoxic ischemic encephalopathy. Journal of Perinatal Medicine, 36(3): 240-247.

15. Chiang M, Lien R, Chu S et al. (2016): Serum lactate, brain magnetic resonance imaging and outcome of neonatal hypoxic ischemic encephalopathy after therapeutic hypothermia. Pediatrics and Neonatology, 57: 35-40.

16. Van Anh T, Hao T, Chi N et al. (2019): Predictions of hypoxic-ischemic encephalopathy by umbilical cord blood lactate in newborns with birth asphyxia. Open Access Macedonian Journal of Medical Sciences, 7(21): 3564.

17. Elfarargy M, Elsharkawy H, Elgendy M (2018): Study of lactate and nucleated red blood cells as early predictors of neonatal hypoxic ischemic encephalopathy. Acta Scientific Paediatrics, 1(2): 3-8.

18. Li J, Kobata K, Kamei Y et al. (2014): Nucleated red blood cell counts: an early predictor of brain injury and 2-year outcome in neonates with hypoxic ischemic encephalopathy in the era of cooling-based treatment. Brain and Development, 36(6): 472-478.

19. Boskabadi H, Rakhshanizadeh $F$, Zakerihamidi $M$ (2020): Assessment of umbilical cord nucleated red blood cell count in discharged and dead very low birth weight infants. Iranian Journal of Neonatology IJN., 11(1): 36-42.

20. Goel M, Dwivedi R, Gohiya P et al. (2013): Nucleated red blood cell in cord blood as a marker of perinatal asphyxia. Journal of Clinical Neonatology, 2(4): 17983. 